\title{
Survival rate of cemented versus cementless tibial component in primary total knee arthroplasty over 5 years of follow-up: comparative study of 109 prostheses
}

\author{
Victor Pacoret ${ }^{1}$, Etienne Kalk $^{2}$, Ludovic Labattut ${ }^{1}$, Guillaume Girardot ${ }^{1}$, Emmanuel Baulot ${ }^{1,3}$, \\ and Pierre Martz ${ }^{1,3, *}$ \\ 1 Dijon University Hospital, Orthopedic and Traumatology Department, 21000 Dijon, France \\ 2 Orthopedic Center, 71640 Dracy-Le-Fort, France \\ 3 INSERM UMR1093-CAPS, Burgundy Franche-Comté University, 21000 Dijon, France
}

Received 2 May 2020, Accepted 28 July 2020, Published online 8 September 2020

\begin{abstract}
Introduction: Knee osteoarthritis is the main indication for primary total knee arthroplasty (TKA). It is now accepted that cementless implantation of the femoral component provides equivalent results to cemented one, however, the optimal fixation method of the tibial component remains controversial. The purpose of this study was to compare the survivorship of cemented versus cementless tibial baseplate in primary total knee arthroplasty. Materials and methods: We carried out a retrospective, monocentric study, including 109 TKA (Zimmer ${ }^{\circledR}$ Natural Knee II ultra-congruent mobile-bearing) implanted between 2004 and 2010 for primary osteoarthritis, comparing 2 groups depending on tibial component fixation method, one cemented $(n=68)$ and one cementless $(n=41)$. Clinical (Knee Society Rating System (KSS), Hospital for Special Surgery (HSS) scores, range of motion) and radiodiological outcomes were assessed at last follow-up with a minimal follow-up of 5 years. Results: Mean follow-up were 8.14 [5.31-12.7] and 8.06 [5.22-12.02] years, respectively, in cemented and cementless groups. The tibial component survival rate was $100 \%$ [95CI: 91.4-100] in the cementless group and 97.1\% [95CI: 89.78-99.42] in the cemented group ( 2 aseptic loosenings) ( $p=0.27)$. Radiolucent lines were present in $31.7 \%(n=13)$ of the cementless and $44.1 \%(n=30)$ of the cemented baseplates $(p=0.2)$. The postoperative KSS knee score was higher in the cementless group ( $99 \pm 3$ vs. $97 \pm 7.5 ; p=0.02$ ), but there was no significant difference in KSS function, global KSS and HSS scores. Mean range of flexion was $120 \pm 10^{\circ}$ in the cementless group and $122.5 \pm 15^{\circ}$ in the cemented group $(p=0.37)$. No significant differences were found on the radiographic data or on complications. Conclusion: In this study, the survival rate of the tibial component is not influenced by its fixation method at a mean follow-up of 8 years in osteoarthritis, which confirms the reliability of cementless fixation in primary TKA.
\end{abstract}

Key words: Total knee arthroplasty, Retrospective, Comparative, Tibia, Cemented, Cementless.

\section{Introduction}

Total knee arthroplasty (TKA) has become the standard treatment for degenerative knee diseases especially in primary osteoarthritis (OA). The implant fixation method, either cemented or cementless, remains controversial, mainly on the tibial side [1-5].

On the one hand, cemented fixation advantages are the instantaneous, spontaneous, and strong primary fixation after acrylic cement polymerization [6], and the possibility to correct a slightly inaccurate bone cut by the cement thickness, with excellent clinical results and survival rate at 20-year follow-up (FU) in several series [7,8], but on the other hand, cementless

\footnotetext{
*Corresponding author: martzpierre@neuf.fr
}

fixation allows mechanical and biological fixation by bony ingrowth on the implant surface, reduction of operating time and an osseointegration that would allow a durable fixation $[2,4,9]$. Outcomes at long-term follow-up are also excellent $[8,10]$.

However, cementless tibial components have a greater micromotion during the first 3 postoperative months, then a stabilization of the component in radiostereometric analysis is achieved, whereas cemented tibial components have an initially lower migration, but over time have a continuously increasing micromotion [11, 12]. Aseptic loosening is one of the main causes of TKA failure, with a predominance on the tibial side [13]. That is why the fixation type of the tibial component should be a crucial factor in the prosthesis survival. 
We hypothesized that cementless tibial fixation in primary osteoarthritis is at least as reliable as cemented fixation at mid-term follow-up. The purpose of our study was to compare survival rate of the tibial component fixation between cemented and cementless TKAs.

\section{Materials and methods}

We performed a retrospective, monocentric study, comparing cementless and cemented fixation of the tibial component on a series of ultra-congruent mobile-bearing Natural Knee II prostheses $\left(\right.$ Zimmer $^{\circledR}$, Warsaw, IN, USA), without patellar resurfacing, implanted in patients suffering from primary osteoarthritis, between 2004 and 2010.

Inclusion criteria were patients suffering from end-stage primary osteoarthritis with an indication of TKA, operated by the same operator (EB), without prior surgery on this knee and with a complete preoperative evaluation. The exclusion criteria were rheumatic disease or other inflammatory arthropathies and an indication for relaxation osteotomy associated with TKA.

A total of 157 patients (184 TKA) were identified. Sixty-six patients were excluded ( 21 died, 30 records were incomplete, 15 patients refused to be reassessed). The analysis included 91 patients and 109 TKA: 41 in the cementless group and 68 in the cemented group.

The preoperative assessment included: a clinical data analysis: age, Body Mass Index (BMI), Devane's activity score [14], University of California in Los Angeles (UCLA) score [15], the Knee Society Rating System (KSS) (knee and function scores) and the Hospital for Special Surgery (HSS) score. A radiographic evaluation included anteroposterior, lateral, and axial views and lower limb telemetry allowing measurement of angles and evaluation of the arthritis stage according to Ahlbäck's original classification [16].

The surgical technique and the implant were the same for all the patients, using a medial para-patellar approach, a cementless femoral component, a cemented or a cementless metal back tibial base CSTi ${ }^{\mathrm{TM}}$ (Cancellous-Structured Titanium), an ultracongruent polyethylene rotating platform, without patellar resurfacing (patelloplasty with patellar decompression). In the case of cemented tibial component, we used a low-viscosity cement impregnated with gentamicin. Rehabilitation was standardized with immediate weight bearing and early knee mobilization. Peroperative data were collected: type of anesthesia (general, spinal anesthesia), operating time, size of the implants, use of a tourniquet, blood loss, ASA (American Society of Anesthesiologists) score.

At the last follow-up visit, all the patients were evaluated by an independent observer experienced in knee arthroplastic surgery, with clinical examination, (KSS and HSS scores). Radiographic evaluation included anteroposterior and lateral views, a $30^{\circ}$ flexion standard skyline view and standing lower-limb telemetry.

The primary outcome was the tibial component loosening defined as: a loosening confirmed by revision surgery, migration of an implant on two successive radiographs, or the appearance of a pathological radiolucent line at the bonecement or bone-implant interface [17].

Secondary outcomes were the postoperative KSS (knee, function) and HSS scores, the postoperative range of motion (ROM), the analysis of radiolucent tibial lines according to Ewald's method [18] (classified in 3 stages according to Ewald's scoring system: $\leq 4$ "probably not significant", 5-9 "should be closely followed for progression", $\geq 10$ "possible or impending failure regardless of symptoms"), the radiographic measurements (HKA, HKS, LDFA, MPTA, Caton-Deschamps index, tibial slope) and the occurrence of complications according to the standardized definitions of the Knee Society [17].

Statistical analysis: Median comparisons were performed using the non-parametric Kruskal-Wallis test and percentage comparisons were performed using the chi-square $\left(\chi^{2}\right)$ test or the Fisher exact method. A value of $p<0.05$ was considered statistically significant. All analyses were performed using STATA v14.0 software.

\section{Results}

Among the 91 patients (109 TKAs) 27 were men (29.7\%) and 64 were women $(70.3 \%)$. Eighteen patients (11 men and 7 women) were operated on both sides $(19.8 \%)$. The mean follow-up was 8.06 [5.22-12.02] years in the cementless group and 8.14 [5.31-12.7] years in the cemented group $(p=0.43)$, with a minimum follow-up over 5 years.

The two groups were comparable without any significant differences on preoperative epidemiological, clinical, and radiographic data. Peroperative data too were not different between the two groups (Table 1).

No loosening occurred in the cementless group and two loosening (one aseptic and one septic) were reported in the cemented group $(2.9 \%)$. The tibial component survival rate was 100\% [95CI: 91.4-100] in the cementless group and 97.1\% [95CI: 89.78-99.42] in the cemented group ( $p=0.27$ ) at a minimum of 5-year follow-up, with no statistically significant difference between the two groups $(p=0.27)$.

The postoperative KSS knee score was higher in the cementless group $(99 \pm 3)$ than in the cemented group $(97 \pm 7.5)$ with a significant difference $(p=0.02)$. When looking at postoperative KSS function and HSS scores, there was no statistically significant difference between the two groups (Table 2).

A radiolucent line was found in at least one Ewald zone on the anteroposterior and/or lateral radiographs in 13 cases of the cementless group at the bone-implant interface $(31.7 \%)$ and 30 cases of the cemented group at the cement-bone interface $(44.1 \%)$ and the difference was not statistically significant $(p=0.2)$. We reported no case of progressive radiolucent line in the cementless group and 1 case in the cemented group $(1.5 \%)$, corresponding to the case of septic loosening with no statistically significant difference $(p=0.44)$.

According to Ewald's score, 5 cases (12.2\%) in the cementless group and 7 cases $(10.3 \%)$ in the cemented group belonged to the category "should be closely followed for progression". One case in the cemented group (1.5\%) was classified as "possible or impending failure regardless of symptoms" and 
Table 1. Epidemiological and intra-operative data.

\begin{tabular}{|c|c|c|c|}
\hline & Cementless group $(n=41)$ & Cemented group $(n=68)$ & $p$ \\
\hline \multicolumn{4}{|l|}{ Epidemiological data } \\
\hline Gender (Men/Women) & $16(39 \%) / 25(61 \%)$ & $22(32 \%) / 46(68 \%)$ & 0.48 \\
\hline Age & $73.7(11.8)$ & $70.3(11.5)$ & 0.29 \\
\hline BMI & $29.4(7.6)$ & $31.1(5.9)$ & 0.72 \\
\hline Meniscectomy & $1(2.4 \%)$ & $8(11.8 \%)$ & 0.09 \\
\hline DEVANE & $4(1)$ & $3(1)$ & 0.33 \\
\hline UCLA & $6(2)$ & $6(2)$ & 0.5 \\
\hline ASA & $2(1)$ & $2(1)$ & 0.31 \\
\hline \multicolumn{4}{|l|}{ Intra-operative data } \\
\hline \multicolumn{4}{|l|}{ Tourniquet } \\
\hline Yes & $9(22 \%)$ & $21(31 \%)$ & 0.31 \\
\hline No & $32(78 \%)$ & $47(69 \%)$ & \\
\hline Spinal anesthesia & $9(22 \%)$ & $13(19 \%)$ & 0.72 \\
\hline General anesthesia & $32(78 \%)$ & $55(81 \%)$ & \\
\hline Operating time (min) & $83.6(21)$ & $90.3(20)$ & 0.06 \\
\hline Blood loss (ml) & $406(300)$ & $367(300)$ & 0.78 \\
\hline
\end{tabular}

BMI: Body Mass Index, UCLA: University of California in Los Angeles score, ASA: American Society of Anesthesiologists score. Variables expressed as means per group with standard deviation.

Table 2. Clinical pre- and postoperative assessments.

\begin{tabular}{lccc}
\hline & $\begin{array}{c}\text { Cementless group } \\
(n=41)\end{array}$ & $\begin{array}{c}\text { Cemented group } \\
(n=68)\end{array}$ & $p$ \\
\hline $\begin{array}{l}\text { Follow-up } \\
\text { (years) }\end{array}$ & $8.06(3.96)$ & $8.14(3.57)$ & 0.43 \\
KSS Knee & {$[5.22-12.02]$} & {$[5.31-12.7]$} & \\
$\quad$ Preoperative & $46(18)$ & $44(17.5)$ & 0.65 \\
$\quad$ Postoperative & $99(3)$ & $97(7.5)$ & $\mathbf{0 . 0 2}$ \\
$\quad$ + Gain & $53(17)$ & $53(15.5)$ & 0.54 \\
KSS Function & & & \\
$\quad$ Preoperative & $50(5)$ & $50(5)$ & 0.38 \\
Postoperative & $100(0)$ & $100(7.5)$ & 0.97 \\
$\quad$ + Gain & $50(10)$ & $50(10)$ & 0.34 \\
KSS Global & & & \\
$\quad$ Preoperative & $94(20)$ & $93.5(21)$ & 0.67 \\
Postoperative & $198(7)$ & $196.5(12)$ & 0.39 \\
$\quad$ + Gain & $101(22)$ & $100(26)$ & 0.44 \\
HSS & & & \\
$\quad$ Preoperative & $64(8)$ & $64(7.5)$ & 0.89 \\
Postoperative & $96(2)$ & $96(4)$ & 0.49 \\
$\quad$ + Gain & $31(9)$ & $31(8)$ & 0.28 \\
Range of motion & & & \\
$\quad$ Preoperative & $115(20)$ & $110(27.5)$ & 0.15 \\
Postoperative & $120(10)$ & $122.5(15)$ & 0.37 \\
\hline
\end{tabular}

KSS: Knee Society Score, HSS: Hospital for Special Surgery. Variables are expressed as means per group with standard deviation into brocket and range into square bracket for the follow-up.

corresponded to the case of septic loosening and progressive line. All other cases $(n=78)$ were classified as "probably not significant". No differences were statistically relevant $(p=0.71)$ (Table 3).

Mean postoperative ROM was $120 \pm 10^{\circ}$ in the cementless group and $122.5 \pm 15^{\circ}$ in the cemented group with no significant difference $(p=0.37)$.
Table 3. Radiographic pre- and postoperative data.

\begin{tabular}{|c|c|c|c|}
\hline & $\begin{array}{c}\text { Cementless group } \\
(n=41)\end{array}$ & $\begin{array}{c}\text { Cemented group } \\
(n=68)\end{array}$ & $p$ \\
\hline \multirow{2}{*}{\multicolumn{4}{|c|}{$\begin{array}{l}\text { Radiographic } \\
\text { measurements } \\
\text { HKA }\end{array}$}} \\
\hline & & & \\
\hline Preoperative & $174(8)$ & $175(9.0)$ & 0.50 \\
\hline Postoperative & $179(3)$ & $178(3.5)$ & 0.05 \\
\hline \multicolumn{4}{|l|}{ LDFA } \\
\hline Preoperative & $91(3)$ & $92(3)$ & 0.35 \\
\hline Postoperative & $89(3)$ & $89(2)$ & 0.34 \\
\hline \multicolumn{4}{|l|}{ MPTA } \\
\hline Preoperative & $88(4)$ & 87 (4) & 0.73 \\
\hline Postoperative & $89(2)$ & $89(3)$ & 0.05 \\
\hline \multicolumn{4}{|l|}{ HKS } \\
\hline Preoperative & $6(1)$ & $6(1)$ & 0.35 \\
\hline Postoperative & $7(1)$ & $6(1)$ & 0.39 \\
\hline \multicolumn{4}{|l|}{ Tibial slope } \\
\hline Preoperative & $86(3)$ & $86(3)$ & 0.28 \\
\hline Postoperative & $86(3)$ & $86(3)$ & 0.2 \\
\hline \multicolumn{4}{|l|}{ CATON index } \\
\hline Preoperative & $0.95(0.15)$ & $0.90(0.15)$ & 0.86 \\
\hline Postoperative & $0.95(0.2)$ & $0.90(0.2)$ & 0.08 \\
\hline Ahlbäck stage & $3(1)$ & $3(1)$ & 0.18 \\
\hline \multicolumn{4}{|l|}{$\begin{array}{l}\text { Radiolucent lines } \\
\text { (RL) }\end{array}$} \\
\hline Standard RL & $13(31.7 \%)$ & $30(44.1 \%)$ & 0.2 \\
\hline Progressive RL & $0(0 \%)$ & $1(1.5 \%)$ & 0.43 \\
\hline \multicolumn{4}{|l|}{ EWALD score } \\
\hline$\leq 4$ & $36(87.8 \%)$ & $60(88.2 \%)$ & \\
\hline $5-9$ & $5(12.2 \%)$ & $7(10.3 \%)$ & 0.71 \\
\hline$\geq 10$ & $0(0 \%)$ & $1(1.5 \%)$ & \\
\hline
\end{tabular}

HKA: Hip Knee Ankle angle, LDFA: Lateral Distal Femoral Angle, MPTA: Medial Proximal Tibial Angle, HKS: Hip Knee Shaft. Variables are expressed as means per group with standard deviation into bracket for radiographic measurements, and number with percentages into brackets for radiolucent lines. 
Table 4. Randomized controlled trials assessing the survival rates of cemented versus cementless tibial component, compared to our study.

\begin{tabular}{llcccc}
\hline Author & Journal & Year & Cemented/Cementless $(n)$ & $\begin{array}{c}\text { Mean } \\
\text { follow-up }\end{array}$ & $\begin{array}{c}\text { Survival rate } \\
\text { Cemented/Cementless }\end{array}$ \\
\hline Nilsson et al. [1] & J Arthroplaty & 1999 & $29 / 28(57)$ & 5 years & $100 \% / 96.3 \%^{*}$ \\
Baker et al. [19] & JBJS & 2007 & $277 / 224(501)$ & 8 years & $95 \% / 94.6 \%^{*}$ \\
Beaupré et al. [2] & JBJS & 2007 & $41 / 40(81)$ & 5 years & $100 \% / 100 \%^{*}$ \\
Dunbar et al. [3] & JBJS & 2009 & $21 / 28(49)$ & 2 years & $100 \% / 100 \%^{*}$ \\
Lizaur-Utrilla et al. [4] & KSSTA & 2014 & $48 / 45(93)$ & 9 years & $90.0 \% / 93.7 \% *$ \\
Choy et al. [5] & J Arthroplasty & 2014 & $86 / 82(168)$ & 9.5 years & $100 \% / 100 \% *$ \\
Kim et al. [10] & Int Ortho & 2014 & $80 / 80(160)$ & 17 years & $100 \% / 98.7 \%^{*}$ \\
This study & & & $68 / 41(109)$ & 8 years & $97.06 \% / 100 \% *$ \\
\hline
\end{tabular}

* Not statistically significant.

There was also no significant difference in blood loss and operating time between the two groups.

Concerning the postoperative radiographic data, no significant difference was reported on lower limb alignment (HKA, LDFA, MPTA, HKS angles, tibial slope, Caton-Deschamps index and patellar position on axial view between the cementless and cemented groups).

Complications listed were one loosening of the femoral component, one peri-prosthetic femoral fracture in the cementless group and one case of polyethylene wear, one patellar dislocation, one septic loosening, one peri-prosthetic femoral fracture, and one patellar tendon rupture in the cemented group.

\section{Discussion}

There was no significant difference in terms of tibial component survival rate between cementless and cemented fixation with a minimum of 5-year postoperative follow-up in our series. This study shows that both fixations, using an ultra-congruent mobile-bearing prosthesis, provide excellent clinical and radiographic outcomes while treating patients suffering from primary osteoarthritis. Only two loosening occurred in the cemented group.

Bone quality differs among the primary diagnosis: in osteoarthritis, bone quality is expected to be higher than in rheumatic diseases where chronic inflammation and long-term corticosteroid therapy usually decrease bone density. Primary stability of implants is crucial for bony ingrowth and cementless component fixation, which is decreased when bone density is low [4]. Cemented fixation should therefore be preferred in cases of low bone quality. We focused on patients with primary osteoarthritis, however, several prospective randomized controlled trials compared tibial component fixation including different diagnoses also found no significant difference between the two fixation methods (Table 4.).

Although, most of the high quality studies reported no survival rate difference between cemented and cementless tibial fixations (Table 4) [1-5, 10, 19-22], recent national registry studies (New Zealand [23] and Australia [24]) found a higher revision rate with cementless component. On the contrary, a meta-analysis of Newman et al. [25] in 2020 reported better survivorship with cementless baseplate.
Though literature remains controversial about tibial component fixation and when a difference is highlighted, it is a slight one. It seems that at short- and mid-term follow-up, cemented fixation provides better survival rate, but at a longer followup cementless tibial fixation could be more efficient. When focusing on specific cohorts, it appears that patients with morbid obesity [26, 27], and men [19], have a higher survival rate with cementless tibial component rather than with cemented one, but those results are not corroborated by larger series.

In this study, only mobile-bearing rotating plates were used. Scientific publications reported no difference in aseptic loosening between mobile and fixed bearing [28]. For some authors, mobile-bearing insert may reduce shear forces at the boneimplant interface, thus reducing micro-movements that are detrimental to the bony ingrowth process of cementless implants.

Radiolucent line was more frequent in the cemented group (44.1\%) than in the cementless group (31.7\%) without significant difference. The literature remains contradictory about it, while Nilsson et al. [1] and Lizaur-Utrilla et al. [4] reported, as we do, more lines on cemented implants, Park and Kim [21] and Choy et al. [5] found more of them on cementless components. For some authors, the radiolucent line in the cemented group should be partly explained by bone necrosis induced by exothermic reaction during acrylic polymerization [29]. Although no difference in survival was found in these studies and ours, progressive radiolucent line is nevertheless recognized as a predictive risk factor for implant loosening [11].

We found substantial clinical benefits (SCB) between all pre- and postoperative clinical scores (SCB recently defined at 39.7 points for KSS knee and 38.6 points for KSS function by Lizaur-Utrilla et al. [30]). The postoperative KSS knee score was 2 points higher in the uncemented group compared to the cemented one and the difference was statistically significant, however, not clinically relevant. Indeed, the minimal clinically important difference (MCID) is 7.2 points for KSS knee according to by Lizaur-Utrilla et al. [30]. In the literature, some series comparing cementless and cemented fixations, report statistically higher KSS knee [4, 9] and function scores [9] in the cementless group, as in the $\mathrm{Hu}$ et al.'s meta-analysis [31] but none of them are higher than the MCID. The other studies did not report significant difference.

There were some limitations in this study that must be considered. Firstly, the retrospective nature of this study and 
the number of patients with a mid-term follow-up should lead to the possibility of selection bias or lack of power. But this a monocentric study, with a single surgeon, and a single prosthesis design, and the same surgical technique for all the patients. The definition used for the survival rate, with a composite outcome could be discussed, but it allows to increase statistical efficiency and to not underestimate the failure rate. Finally, the radiographs were not fluoroscopically guided, so it could lead to mistakes while assessing radiolucent lines, due to malpositioning of the X-ray.

\section{Conclusion}

Cementless tibial fixation was found as reliable as cemented fixation, with a Natural Knee II (Zimmer ${ }^{\circledR}$, Warsaw, IN, USA) TKA with an ultra-congruent mobile-bearing rotating platform, in patients with primary osteoarthritis, at a minimum of 5-year follow-up. There was no statistically significant difference between the two tibial fixations on survival rate and function at mid-term follow-up.

\section{Conflict of interest}

All the authors certify that he or she has no financial conflict of interest (e.g., consultancies, stock ownership, equity interest, patent/licensing arrangements, etc.) in connection with this article.

\section{IRB/Ethical Committee approval}

After consulting the local ethics committee (CPP est 1. Dijon. France) this routine care retrospective study, in accordance with current French legislation at the time of the inclusion, did not require any ethic committee approbation

\section{References}

1. Nilsson KG, Kärrholm J, Carlsson L, Dalén T (1999) Hydroxyapatite coating versus cemented fixation of the tibial component in total knee arthroplasty. J Arthroplasty 14, 9-20.

2. Beaupré LA, al-Yamani M, Huckell JR, Johnston DWC (2007) Hydroxyapatite-coated tibial implants compared with cemented tibial fixation in primary total knee arthroplasty: a randomized trial of outcomes at five years. J Bone Jt Surg 89, 2204-2211.

3. Dunbar M, Wilson DAJ, Hennigar A, et al. (2009) Fixation of a trabecular metal knee arthroplasty component: a prospective randomized study. J Bone Jt Surg Am 91, 1578-1586.

4. Lizaur-Utrilla A, Miralles-Muñoz FA, Lopez-Prats FA (2014) Similar survival between screw cementless and cemented tibial components in young patients with osteoarthritis. Knee Surg Sports Traumatol Arthrosc 22, 1585-1590.

5. Choy W-S, Yang D-S, Lee K-W, et al. (2014) Cemented versus cementless fixation of a tibial component in LCS mobilebearing total knee arthroplasty performed by a single surgeon. J Arthroplasty 29, 2397-2401.

6. Cawley DT, Kelly N, McGarry JP, Shannon FJ (2013) Cementing techniques for the tibial component in primary total knee replacement. Bone Jt J 95-B, 295-300.
7. Ritter MA, Keating EM, Sueyoshi T, et al. (2016) Twenty-fiveyears and greater, results after nonmodular cemented total knee arthroplasty. J Arthroplasty 31, 2199-2202.

8. Long WJ, Bryce CD, Hollenbeak CS, et al. (2014) Total knee replacement in young, active patients: long-term follow-up and functional outcome: a concise follow-up of a previous report. $\mathrm{J}$ Bone Jt Surg Am 96, e159-1-e159-7.

9. Bassett RW (1998) Results of 1,000 performance knees: cementless versus cemented fixation. J Arthroplasty 13, 409-413.

10. Kim Y-H, Park J-W, Lim H-M, Park E-S (2014) Cementless and cemented total knee arthroplasty in patients younger than fifty five years. Which is better? Int Orthop 38, 297-303.

11. van Hamersveld KT, Marang-van de Mheen PJ, Tsonaka R, et al. (2017) Fixation and clinical outcome of uncemented periapatite-coated versus cemented total knee arthroplasty: five-year follow-up of a randomised controlled trial using radiostereometric analysis (RSA). Bone Jt J 99-B, 1467-1476.

12. Laende EK, Richardson CG, Dunbar MJ (2019) Predictive value of short-term migration in determining long-term stable fixation in cemented and cementless total knee arthroplasties. Bone Jt J 101-B, 55-60.

13. Sharkey PF, Lichstein PM, Shen C, et al. (2014) Why are total knee arthroplasties failing today - Has anything changed after 10 years? J Arthroplasty 29, 1774-1778.

14. Devane PA, Horne JG, Martin K, et al. (1997) Threedimensional polyethylene wear of a press-fit titanium prosthesis. Factors influencing generation of polyethylene debris. J Arthroplasty 12, 256-266.

15. Zahiri CA, Schmalzried TP, Szuszczewicz ES, Amstutz HC (1998) Assessing activity in joint replacement patients. J Arthroplasty 13, 890-895.

16. Ahlbäck S (1968) Osteoarthrosis of the knee. A radiographic investigation. Acta Radiol Diagn (Stockh) Suppl 277, 7-72.

17. Healy WL, Della Valle CJ, Iorio R, et al. (2013) Complications of total knee arthroplasty: standardized list and definitions of the Knee Society. Clin Orthop Relat Res 471, 215-220.

18. Ewald FC (1989) The Knee Society total knee arthroplasty roentgenographic evaluation and scoring system. Clin Orthop 248, 9-12.

19. Baker PN, Khaw FM, Kirk LMG, et al. (2007) A randomised controlled trial of cemented versus cementless press-fit condylar total knee replacement: 15-year survival analysis. J Bone Jt Surg 89, 1608-1614.

20. Carlsson Å, Björkman A, Besjakov J, Önsten I (2005) Cemented tibial component fixation performs better than cementless fixation: A randomized radiostereometric study comparing porous-coated, hydroxyapatite-coated and cemented tibial components over 5 years. Acta Orthop 76, 362-369.

21. Park J-W, Kim Y-H (2011) Simultaneous cemented and cementless total knee replacement in the same patients: A prospective comparison of long-term outcomes using an identical design of NexGen prosthesis. J Bone Joint Surg Br 93-B, 1479-1486.

22. Fernandez-Fairen M, Hernández-Vaquero D, Murcia A, et al. (2013) Trabecular metal in total knee arthroplasty associated with higher knee scores: a randomized controlled trial. Clin Orthop Relat Res 471, 3543-3553.

23. Nugent M, Wyatt MC, Frampton CM, Hooper GJ (2019) Despite improved survivorship of uncemented fixation in total knee arthroplasty for osteoarthritis, cemented fixation remains the gold standard: an analysis of a National Joint Registry. J Arthroplasty 34, 1626-1633. 
24. Jorgensen NB, McAuliffe M, Orschulok T, et al. (2019) Major aseptic revision following total knee replacement: a study of 478,081 total knee replacements from the Australian Orthopaedic Association National Joint Replacement Registry. J Bone Jt Surg 101, 302-310.

25. Newman JM, Sodhi N, Dekis JC, et al. (2020) Survivorship and functional outcomes of cementless versus cemented total knee arthroplasty: a meta-analysis. J Knee Surg 33, 270-278.

26. Sinicrope BJ, Feher AW, Bhimani SJ, et al. (2019) Increased survivorship of cementless versus cemented tka in the morbidly obese. A minimum 5-year follow-up. J Arthroplasty 34, 309-314.

27. Bagsby DT, Issa K, Smith LS, et al. (2016) Cemented vs cementless total knee arthroplasty in morbidly obese patients. J Arthroplasty 31, 1727-1731.
28. Kim Y-H, Park J-W, Kim J-S (2019) The long-term results of simultaneous high-flexion mobile-bearing and fixed-bearing total knee arthroplasties performed in the same patients. J Arthroplasty 34, 501-507.

29. Wimhurst JA, Brooks RA, Rushton N (2001) The effects of particulate bone cements at the bone-implant interface. J Bone Jt Surg 83, 5 .

30. Lizaur-Utrilla A, Gonzalez-Parreño S, Martinez-Mendez D, et al. (2020) Minimal clinically important differences and substantial clinical benefits for Knee Society scores. Knee Surg Sports Traumatol Arthrosc 28, 1473-1478.

31. Hu B, Chen Y, Zhu H, et al. (2017) Cementless porous tantalum monoblock tibia vs cemented modular tibia in primary total knee arthroplasty: a meta-analysis. J Arthroplasty 32, 666-674.

Cite this article as: Pacoret V, Kalk E, Labattut L, Girardot G, Baulot E \& Martz P (2020) Survival rate of cemented versus cementless tibial component in primary total knee arthroplasty over 5 years of follow-up: comparative study of 109 prostheses. SICOT-J 6, 36 